\title{
Baseline Sensitivity of Pyraclostrobin and Toxicity of SHAM to Sclerotinia sclerotiorum
}

Hong-Jie Liang, Ya-Li Di, Jin-Li Li, Hong You, and Fu-Xing Zhu, College of Plant Science and Technology, Huazhong Agricultural University, Wuhan, 430070, China

\begin{abstract}
Liang, H.-J., Di, Y.-L., Li, J.-L., You, H., and Zhu, F.-X. 2015. Baseline sensitivity of pyraclostrobin and toxicity of SHAM to Sclerotinia sclerotiorum. Plant Dis. 99:267-273.

Sclerotinia sclerotiorum is a cosmopolitan plant pathogen notable for its wide host range. The quinone outside inhibitor (QoI) fungicide pyraclostrobin has not been registered for control of $S$. sclerotiorum in China. In this study, baseline sensitivity of pyraclostrobin was established based on effective concentration for $50 \%$ inhibition of mycelial growth $\left(\mathrm{EC}_{50}\right)$ values of 153 isolates of $S$. sclerotiorum collected from five provinces of China and toxicity of alternative oxidase inhibitor salicylhydroxamic acid (SHAM) to S. sclerotiorum was determined. Results showed that the frequency distribution of $\mathrm{EC}_{50}$ values of the 153 isolates was unimodal but with a right-hand tail. The mean $\mathrm{EC}_{50}$ value was $0.1027 \mu \mathrm{g} / \mathrm{ml}$ and the range of $\mathrm{EC}_{50}$ values was 0.0124 to

pyraclostrobin at 5,15 , and $45 \mu \mathrm{g} / \mathrm{ml}$ provided control efficacies of 61 , 77 , and $100 \%$, respectively. There was no positive cross-resistance between pyraclostrobin and carbendazim or dimethachlon. $\mathrm{EC}_{50}$ values for SHAM against four isolates of $S$. sclerotiorum were $44.4,51.8$, 54.4 , and $68.7 \mu \mathrm{g} / \mathrm{ml}$. SHAM at $20 \mu \mathrm{g} / \mathrm{ml}$ could significantly increase not only the inhibitory effect of pyraclostrobin on mycelial growth on potato dextrose agar media but also the control efficacy in planta. These results indicated that SHAM should not be added into artificial media in in vitro assay of $S$. sclerotiorum sensitivity to pyraclostrobin. This has broad implications for assay of sensitivity of fungal pathogen to QoI fungicides.
\end{abstract} $0.6324 \mu \mathrm{g} / \mathrm{ml}$. Applied as a preventive fungicide in pot experiments,
The ascomycete fungus Sclerotinia sclerotiorum (Lib.) de Bary is a highly destructive plant pathogen capable of infecting more than 400 species of plants worldwide, including economically important crops and vegetables such as oilseed rape, soybean, sunflower, peanut, cabbage, cauliflower, and tomato $(4,5)$. It was reported that the annual losses caused by $S$. sclerotiorum in the United States exceeded U.S.\$200 million in the late 20th century (5). In Jiangsu Province of eastern China, yield losses of oilseed rape caused by this pathogen range from 10 to $80 \%$ (33). Diseases caused by $S$. sclerotiorum have traditionally been difficult to control because resistant cultivars have not yet been sufficiently developed in major crops. In practice, application of fungicides is still the principal method for control of S. sclerotiorum in oilseed rape in China (33). The benzimidazole fungicide carbendazim was extensively used to control S. sclerotiorum during the 1980 s and 1990 s in China. As a result, high levels of resistance to carbendazim were reported in the late 1990s and became widespread in eastern China in the following years $(22,29,39)$. Mainly due to field control failures caused by the development of resistance to carbendazim, the dicarboximide fungicides dimethachlon, iprodione, and procymidone began to be commonly used for control of S. sclerotiorum around the 2000s in China. More than a decade of dimethachlon applications resulted in low levels of resistance to dimethachlon in Jiangsu Province of eastern China (19). More recently, medium to high levels of resistance to dimethachlon have been reported in Shaanxi Province of northwestern China, Hunan Province of central China, and Heilongjiang Province of northeastern China. Furthermore, there was positive crossresistance among different members of the dicarboximide fungicide group $(40,41)$. With the development of resistance to traditionally used fungicides, it is important and urgent to find

Corresponding author: F.-X. Zhu, E-mail: zhufuxing@mail.hzau.edu.cn

Accepted for publication 28 August 2014.

http://dx.doi.org/10.1094/PDIS-06-14-0633-RE

(C) 2015 The American Phytopathological Society proper substitute fungicides for successful management of $S$. sclerotiorum.

The quinone outside inhibitor (QoI) fungicides are the most important class of agricultural fungicides in respect to market share and sales. The discovery of this class of fungicides was based on a group of natural products synthesized by wood-rotting fungi, including one called Strobilurus tenacellus. Hence, the QoI fungicides are also referred to as the strobilurins in recognition of the source of the lead compounds for development of this class of fungicides (31). The biochemical mode of action of QoI fungicides is to block electron transport at the quinol-oxidizing site of the cytochrome bc1 complex (complex III) in the mitochondrial respiration chain, thus halting synthesis of adenosine triphosphate. The QoI fungicides are effective in inhibiting sporulation, spore germination, and mycelial growth of the target fungal pathogens. Because of their broad-spectrum activity, QoI fungicides have been widely used in agriculture $(3,34)$. Pyraclostrobin belongs to the QoI class and was developed and commercialized by BASF Corporation (Ludwigshafen, Germany) in the early 2000s. It was reported that pyraclostrobin has excellent preventive, curative, and eradicative activities against a wide range of plant pathogens (38). Thus far, pyraclostrobin has not yet been registered in China on oilseed rape or soybean for control of S. sclerotiorum. The only registered QoI fungicide for control of $S$. sclerotiorum in China was tricyclopyricarb (China Pesticide Information Network, http://www.chinapesticide.gov.cn). At present, no case of S. sclerotiorum resistance to QoI fungicide has been reported in China.

Establishment of baseline sensitivity before introduction of a new fungicide into market is essential for subsequent resistance monitoring programs and is required during the registration process in the European Union (27). It has been reported that fungi may avoid the fungitoxic effects of QoI fungicides through the alternative oxidase (AOX) located on the mitochondrial membrane whereas, during fungal infection of plants, AOX is presumed to be inhibited by plant secondary metabolites such as flavonoid $(13,35)$. Therefore, an AOX characteristic inhibitor, salicylhydroxamic acid (SHAM), is commonly included in artificial culture media to suppress the alternative respiration $(9,20,21)$. In most cases, the addition of SHAM, usually at $100 \mu \mathrm{g} / \mathrm{ml}$, will reduce effective concen- 
tration for $50 \%$ inhibition of mycelial growth $\left(\mathrm{EC}_{50}\right)$ values for the tested QoI fungicide, as demonstrated in Ascochyta rabiei (34) and S. sclerotiorum (9). However, the dose response to QoI fungicide in the presence of SHAM may not always represent the real sensitivity to the tested fungicide for some fungal plant pathogens. For example, $\mathrm{EC}_{50}$ value for the QoI fungicide azoxystrobin in the presence of SHAM against Fusicladium effusum was higher, rather than lower, than that for azoxystrobin alone (28). The $\mathrm{EC}_{50}$ values of the sensitive isolates of Microdochium nivale were also higher for azoxystrobin in the presence of SHAM $(0.5 \mathrm{mM})$ compared with azoxystrobin without SHAM (32). The sole purpose for addition of SHAM into artificial media during in vitro assay of QoI fungicide sensitivity is to inhibit AOX. However, these results indicate that the inhibition of AOX is not the only effect of SHAM on plant pathogens; thus, the $\mathrm{EC}_{50}$ values of $\mathrm{QoI}$ fungicide in the presence of SHAM may overestimate or underestimate the real QoI fungicide sensitivity. This is consistent with the report that the biological activity of SHAM on plant pathogens is pathogen dependent (35). Whether SHAM should be added into artificial media in in vitro assay of pyraclostrobin sensitivity in S. sclerotiorum deserves an investigation. Therefore, the objectives of this study were to (i) establish the baseline sensitivity of S. sclerotiorum to pyraclostrobin, (ii) assess preventive and curative activities of pyraclostrobin against $S$. sclerotiorum, and (iii) investigate the toxic effect and role of SHAM in assay of S. sclerotiorum sensitivity to pyraclostrobin.

\section{Materials and Methods}

Origins and collections of $S$. sclerotiorum isolates. Isolates $(N$ $=153$ ) of $S$. sclerotiorum were collected in 2013 from the following hosts and regions: 62 isolates from oilseed rape fields in Hubei and Hunan Provinces of central China, 62 isolates from oilseed rape fields in Gansu and Shaanxi Provinces of northwestern China, and 29 isolates from soybean fields in Heilongjiang Province of northeastern China. The collection fields were separated from one another by more than $20 \mathrm{~km}$. In each field, several plants with typical symptoms of Sclerotinia stem rot were arbitrarily selected. Sclerotia collected from the same infected stem of oilseed rape or soybean plant were regarded as one isolate.

Fungicides. All fungicides were technical-grade products. Pyraclostrobin (95\% active ingredient [a.i.]) was provided by Hubei Kang Bao Tai Fine-Chemical Co. Ltd. and dissolved in acetone to produce a $1,000-\mu \mathrm{g} / \mathrm{ml}$ stock solution. Dimethachlon $(96.2 \%$ a.i.) was provided by Wenzhou Pesticide Co. Ltd. and dissolved in methanol to produce a $1,000-\mu \mathrm{g} / \mathrm{ml}$ stock solution. Carbendazim ( $98.1 \%$ a.i.) was provided by Haili Guixi Chemical Co. Ltd. and dissolved in hydrochloric acid $(\mathrm{HCl})$ at 0.1 mole/liter to produce a $1,000 \mu \mathrm{g} / \mathrm{ml}$ stock solution. The stock solutions were stored in a refrigerator at $4^{\circ} \mathrm{C}$ for no longer than 2 weeks before being serially diluted for bioassay experiments.

Determination of $\mathbf{E C}_{50}$ values and baseline sensitivity of $S$. sclerotiorum to pyraclostrobin. Determination of the $\mathrm{EC}_{50}$ was performed according to Ma et al. (19), with minor modifications. Briefly, potato dextrose agar (PDA) media were routinely amended with pyraclostrobin at final concentrations of $0.0125,0.025,0.05$, $0.1,0.2$, and $0.4 \mu \mathrm{g}$ a.i./ml. For some isolates, one or two extra concentrations of $0.003125,0.00625$, or $0.8 \mu \mathrm{g}$ a.i. $/ \mathrm{ml}$ were used to ensure that, for each isolate, there was at least one concentration of pyraclostrobin having a mycelial growth percent inhibition greater than $50 \%$ and, at the same time, at least another concentration having a percent inhibition lower than 50\%. Mycelial plugs $(6 \mathrm{~mm}$ in diameter) cut from the periphery of 2-day-old colonies of each isolate were placed upside down onto the center of fungicideamended PDA plates. The fungicide-free PDA plates were used as the control. PDA plates were incubated at $26^{\circ} \mathrm{C}$ for $48 \mathrm{~h}$ in the dark. The diameter of each colony was measured twice at right angles. The experiments were performed in triplicate. Pyraclostrobin concentrations and their respective percent inhibitions were used to calculate $\mathrm{EC}_{50}$ values by the procedure of Bioassay Analysis for Quantitative Data in Data Processing System (ver.
7.05; Hangzhou RuiFeng Information Technology Co. Ltd.). Baseline sensitivity of pyraclostrobin was constructed based on frequency distributions of $\mathrm{EC}_{50}$ values of the 153 isolates.

Determination of cross-resistance between pyraclostrobin and carbendazim or dimethachlon. Three dimethachlon-resistant isolates from Heilongjiang Province, three carbendazim-resistant isolates from Hunan Province, and three dual-sensitive isolates (sensitive to both dimethachlon and carbendazim) from Hunan, Gansu, and Heilongjiang Provinces were used to determine the pattern of cross-resistance among pyraclostrobin, dimethachlon, and carbendazim. $\mathrm{EC}_{50}$ values of the nine isolates for the three fungicides were assayed as described above. Serial concentrations of dimethachlon and carbendazim were prepared so that, for each isolate, there was at least one concentration with a percent inhibition above $50 \%$ and at least another concentration with a percent inhibition below $50 \%$. Correlation coefficients $(r)$ between sensitivity to pyraclostrobin and that to dimethachlon and carbendazim were calculated by the correlation procedure in GraphPad Prism (version 5.01; GraphPad Software, Inc.) from the logarithmically transformed $\mathrm{EC}_{50}$ values.

Preventive and curative activities of pyraclostrobin against S. sclerotiorum on detached leaves of oilseed rape. Leaves of similar size (12 to $15 \mathrm{~cm}$ in diameter) were detached from 7- to 8week-old oilseed rape plants, rinsed with sterile water, air dried, and transferred to $15-\mathrm{cm}$ petri dishes lined with wet filter paper to maintain high humidity. Pyraclostrobin solutions were prepared by diluting pyraclostrobin stock solution with $0.1 \%$ Triton X-100 in water. Detached leaves were sprayed with pyraclostrobin solutions at 10,20 , and $40 \mu \mathrm{g} / \mathrm{ml}$ by a hand-held sprayer (Xinmeir Co. Ltd.). Carbendazim was used as the reference fungicide. The treated leaves were air dried for $1 \mathrm{~h}$, then inoculated on the adaxial surface with inverted mycelial plugs cut from actively growing margins of 2-day-old colonies on PDA media. The inoculated leaves were incubated in a growth chamber at $26^{\circ} \mathrm{C}$. Lesion diameters were measured twice at right angles after 24 and $48 \mathrm{~h}$ of incubation. For determination of curative activity, the leaves were inoculated with mycelial plugs and incubated for $24 \mathrm{~h}$, then sprayed with fungicide dilutions as described above. Lesion diameters were measured twice at right angles 24 and $48 \mathrm{~h}$ after fungicide application. The experiments were performed in quadruplicate.

Preventive and curative activities of pyraclostrobin against S. sclerotiorum in pot experiments. Preventive and curative activities of pyraclostrobin on potted oilseed rape plants were assessed with a similar procedure as described above. Seven- to eight-week-old oilseed rape plants growing in 20-cm-diameter pots containing a composite mixture of peat and soil (weight ratio, 1:1) in the greenhouse at $26 \pm 1^{\circ} \mathrm{C}$ were used to substitute for detached leaves. The concentrations of pyraclostrobin and carbendazim were 5,15 , and $45 \mu \mathrm{g} / \mathrm{ml}$ with the $0.1 \%$ Triton $\mathrm{X}-100$ in water as the control. Oilseed rape plants were sprayed with fungicide solutions until runoff. Lesion diameters were measured twice at right angles after 48 and $72 \mathrm{~h}$ of incubation for preventive activity, and 48 and $72 \mathrm{~h}$ after fungicide application for curative activity. The experiments were arranged in a completely randomized design with four replicate oilseed rape plants for each treatment.

Toxic effects of SHAM on mycelial growth of S. sclerotiorum on PDA. Technical-grade product of SHAM (99\% a.i.; SigmaAldrich) was dissolved and serially diluted with methanol to obtain final concentrations of $10,20,40,80,160$, and $320 \mu \mathrm{g} / \mathrm{ml}$ in PDA media. PDA amended with only methanol at a final concentration of $0.2 \%$ ( $\mathrm{vol} / \mathrm{vol}$ ) was used as the control. Mycelial plugs $(6 \mathrm{~mm}$ in diameter) cut from the periphery of 2-day-old colonies of 10 isolates with pyraclostrobin $\mathrm{EC}_{50}$ values lower than $0.1 \mu \mathrm{g} / \mathrm{ml}$ were placed upside down onto the center of SHAM-amended PDA plates. PDA plates were incubated at $26^{\circ} \mathrm{C}$ for $48 \mathrm{~h}$ in the dark. The diameter of each colony was measured twice at right angles. The experiments were performed in triplicate.

Similarly, in determination of $\mathrm{EC}_{50}$ values for pyraclostrobin in the presence of SHAM, the stock solution of SHAM was added to a duplicate set of pyraclostrobin-amended PDA to provide a final 
concentration of SHAM at $20 \mu \mathrm{g} / \mathrm{ml}$. PDA amended with methanol at a final concentration of $0.2 \%(\mathrm{vol} / \mathrm{vol})$ was used as the control. Measurements of mycelial growth and subsequent calculations of $\mathrm{EC}_{50}$ values were the same as described above for pyraclostrobin without SHAM.

Potentiation of control efficacy of pyraclostrobin by SHAM in pot experiments. The experiment procedure was similar to that for determination of preventive control efficacy of pyraclostrobin in pot experiments, as described above. Stock solution of SHAM (dissolved in methanol at $10,000 \mu \mathrm{g} / \mathrm{ml}$ ) was diluted with $0.1 \%$ Triton X-100 in water to get a final concentration of $20 \mu \mathrm{g} / \mathrm{ml}$. The mixture solution of pyraclostrobin and SHAM (at final concentrations of 10 and $20 \mu \mathrm{g} / \mathrm{ml}$, respectively) was prepared accordingly. Control efficacies of SHAM at $20 \mu \mathrm{g} / \mathrm{ml}$, pyraclostrobin at 10 $\mu \mathrm{g} / \mathrm{ml}$, and a mixture of pyraclostrobin and SHAM after $72 \mathrm{~h}$ of incubation were calculated as described above. Co-toxicity factor was calculated according to Kosman and Cohen (16) with the formula co-toxicity factor $=$ (control efficacy of mixture - theoretical control efficacy of mixture)/theoretical control efficacy of mixture, where theoretical control efficacy of mixture $=$ control efficacy of SHAM + control efficacy of pyraclostrobin - control efficacy of SHAM $\times$ control efficacy of pyraclostrobin.

Statistical analysis. Control efficacies were calculated by the following formula. The diameter of mycelial plugs was subtracted from the measured lesion diameters before calculating control efficacies: control efficacy $(\%)=[$ (lesion diameter of the control lesion diameter of the treated)/lesion diameter of the control] $\times 100$.

The procedure of Tukey's multiple comparison test of analysis of variance in GraphPad Prism (version 5.01; GraphPad Software, Inc.) was used to test significant differences in the mean $\mathrm{EC}_{50}$ values between different $S$. sclerotiorum populations. Student's $t$ test was employed to compare control efficacy under the same conditions between carbendazim and pyraclostrobin. Control efficacy data were arcsine square-root transformed before statistical analysis. $\mathrm{EC}_{50}$ data were logarithmically transformed for analysis of correlation between sensitivity to different fungicides.

\section{Results}

Baseline sensitivity of $S$. sclerotiorum to pyraclostrobin. The mean $\mathrm{EC}_{50}$ values for the five populations of S. sclerotiorum collected from different provinces of China were very similar to one another. There was no significant difference $(P=0.9758)$ in mean $\mathrm{EC}_{50}$ values among the five populations (Table 1). Therefore, individual $\mathrm{EC}_{50}$ values for the 153 isolates were pooled together to construct a combined sensitivity baseline for isolates from different regions of China. The frequency distribution of the $\mathrm{EC}_{50}$ values was a unimodal curve, albeit with a long right-hand tail (Fig. 1). The mean $\mathrm{EC}_{50}$ value for the 153 isolates of $S$. sclerotiorum was $0.1027 \mu \mathrm{g} / \mathrm{ml}$ and the range of $\mathrm{EC}_{50}$ values was 0.0124 to 0.6324 $\mu \mathrm{g} / \mathrm{ml}$ (Table 1).

Cross-resistance between pyraclostrobin and carbendazim, dimethachlon. The correlation coefficient $(r)$ between $\mathrm{EC}_{50}$ values for pyraclostrobin and those for dimethachlon was 0.282 while $r=$
-0.476 for pyraclostrobin and carbendazim (Fig. 2). None of the correlations was statistically significant $(P=0.462$ and 0.195 , respectively) and, therefore, no positive or negative cross-resistance between pyraclostrobin and either dimethachlon or carbendazim was detected.

Preventive and curative activities of pyraclostrobin against S. sclerotiorum on detached leaves of oilseed rape. Experiments on detached leaves showed that pyraclostrobin provided higher preventive control efficacies than reference fungicide carbendazim, and the curative control efficacies for the two fungicides were lower than their respective preventive control efficacies (Table 2). Pyraclostrobin applied at $10 \mu \mathrm{g} / \mathrm{ml} 1 \mathrm{~h}$ before inoculation provided 93\% reduction in disease severity whereas preventive control efficacy of carbendazim at the same concentration was only $45 \%$. In regards to curative efficacy, pyraclostrobin applied at $10 \mu \mathrm{g} / \mathrm{ml} 24 \mathrm{~h}$ after inoculation exhibited fungicidal activities similar to those of carbendazim.

Preventive and curative activities of pyraclostrobin against S. sclerotiorum in pot experiments. Control efficacies on potted oilseed rape plants showed that preventive fungicidal activities of pyraclostrobin at 5 and $45 \mu \mathrm{g} / \mathrm{ml} 72 \mathrm{~h}$ after inoculation were significantly higher $(P<0.05)$ than those of carbendazim (Table 3$)$. However, at a concentration of $15 \mu \mathrm{g} / \mathrm{ml}$, the difference was not significant $(P>0.05)$ between the two fungicides. At all three concentrations, the curative activities of pyraclostrobin were similar to those of carbendazim. Compared with preventive activities, the curative activities of pyraclostrobin were significantly lower $(P<$ $0.05)$. Pyraclostrobin at $45 \mu \mathrm{g} / \mathrm{ml}$ provided only $50 \%$ curative control efficacy whereas the preventive control efficacy at the same concentration was $100 \%$.

Toxic effects of SHAM on mycelial growth of S. sclerotiorum on PDA media. $\mathrm{EC}_{50}$ values for SHAM against four S. scleroti-

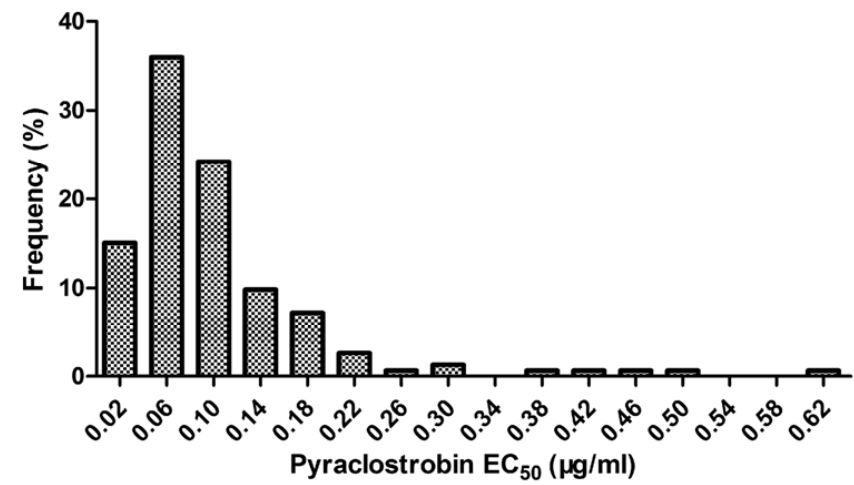

Fig. 1. Frequency distribution of pyraclostrobin sensitivity (effective concentration for $50 \%$ inhibition of mycelial growth $\left[\mathrm{EC}_{50}\right.$ ] values) of 153 isolates of Sclerotinia sclerotiorum collected from different regions of China. Individual $\mathrm{EC}_{50}$ values were grouped in class intervals of $0.04 \mu \mathrm{g} / \mathrm{ml}$. Values on the X-axis indicate the midpoint of the interval.

Table 1. Pyraclostrobin sensitivity in five populations of Sclerotinia sclerotiorum isolates collected from different regions of China

\begin{tabular}{|c|c|c|c|c|c|}
\hline \multirow[b]{2}{*}{ Host } & \multirow[b]{2}{*}{ Collection region } & \multirow[b]{2}{*}{$N^{\mathbf{y}}$} & \multicolumn{3}{|c|}{$\mathbf{E C}_{50}(\mu \mathrm{g} / \mathrm{ml})^{\mathbf{x}}$} \\
\hline & & & Range & Mean \pm standard deviation & Significance $^{\mathrm{x}}$ \\
\hline \multirow[t]{4}{*}{ Oilseed rape } & Hubei & 32 & $0.0249-0.3090$ & $0.1110 \pm 0.0660$ & $\mathrm{a}$ \\
\hline & Hunan & 30 & $0.0252-0.6324$ & $0.1048 \pm 0.1268$ & $\mathrm{a}$ \\
\hline & Gansu & 30 & $0.0336-0.2861$ & $0.1009 \pm 0.0506$ & $\mathrm{a}$ \\
\hline & Shaanxi & 32 & $0.0156-0.5138$ & $0.1005 \pm 0.1297$ & $\mathrm{a}$ \\
\hline Soybean & Heilongjiang & 29 & $0.0124-0.1973$ & $0.0955 \pm 0.0511$ & $\mathrm{a}$ \\
\hline Total & $\ldots$ & 153 & $0.0124-0.6324$ & $0.1027 \pm 0.0916$ & $\ldots$ \\
\hline
\end{tabular}

${ }^{\mathrm{x}}$ Determination of effective concentration for $50 \%$ inhibition of mycelial growth $\left(\mathrm{EC}_{50}\right)$ values was based on inhibition of mycelial radial growth on PDA media.

y Number of isolates.

${ }^{\mathrm{z}}$ Tukey's multiple comparison test of analysis of variance procedure in GraphPad Prism (ver. 5.01) at $P=0.05$ was employed for multiple comparisons between different populations of isolates. The same letter in the column indicates there was no significant difference in the mean $\mathrm{EC}_{50}$ values at $5 \%$ significance level. 
orum isolates (HN26, HB17, HB4, and GS10) were 44.40, 51.80, 54.43 , and $68.68 \mu \mathrm{g} / \mathrm{ml}$, respectively. No inhibitory effects on mycelial growth on PDA media were observed for SHAM at a final concentration of $10 \mu \mathrm{g} / \mathrm{ml}$ (Fig. 3). SHAM at $40 \mu \mathrm{g} / \mathrm{ml}$ could significantly inhibit mycelial growths of all four isolates. These results indicated that SHAM at a concentration equal to or greater than $20 \mu \mathrm{g} / \mathrm{ml}$ had apparent toxic effects on mycelial growth of $S$. sclerotiorum on PDA media.

Potentiation of pyraclostrobin in vitro toxicity and control efficacy by SHAM. EC $_{50}$ values for pyraclostrobin were greatly reduced by addition of SHAM into PDA media. Of the 10 tested isolates, individual $\mathrm{EC}_{50}$ values of 7 isolates for pyraclostrobin with SHAM at $20 \mu \mathrm{g} / \mathrm{ml}$ were significantly lower than their respective $\mathrm{EC}_{50}$ values for pyraclostrobin without SHAM (Table 4). The average $\mathrm{EC}_{50}$ value of the 10 isolates for pyraclostrobin without SHAM was $0.0587 \mu \mathrm{g} / \mathrm{ml}$ whereas the average $\mathrm{EC}_{50}$ value for pyraclostrobin with SHAM was $0.0173 \mu \mathrm{g} / \mathrm{ml}$. Taking the 10 isolates as a group, a paired $t$ test showed that the difference in the mean $\mathrm{EC}_{50}$ values was extremely significant $(P<0.0001)$ between pyra- clostrobin with and without SHAM. Similarly, SHAM at a concentration of $20 \mu \mathrm{g} / \mathrm{ml}$ greatly increased control efficacies of pyraclostrobin against three of the four tested isolates in pot experiments. The co-toxicity factors for SHAM and pyraclostrobin against isolate HN18, HN26, and SX23 were higher than 67 (Table 5). However, for control of isolate HB4, antagonism was detected between SHAM and pyraclostrobin.

\section{Discussion}

Baseline sensitivity data are valuable for resistance risk assessment. The wide range of the baseline $\mathrm{EC}_{50}$ values for pyraclostrobin and especially the long right-hand tail on the sensitivity distribution curve (Fig. 1) may imply a high risk for resistance development in the future although, at present, no definite correlation can be established between the range of sensitivity distribution and the risk of resistance development (7). The site-specific mode of action of pyraclostrobin also indicates a high potential for development of resistance, as do QoI fungicides in general. In most cases, only one point mutation in the mitochondrial cytochrome $b$
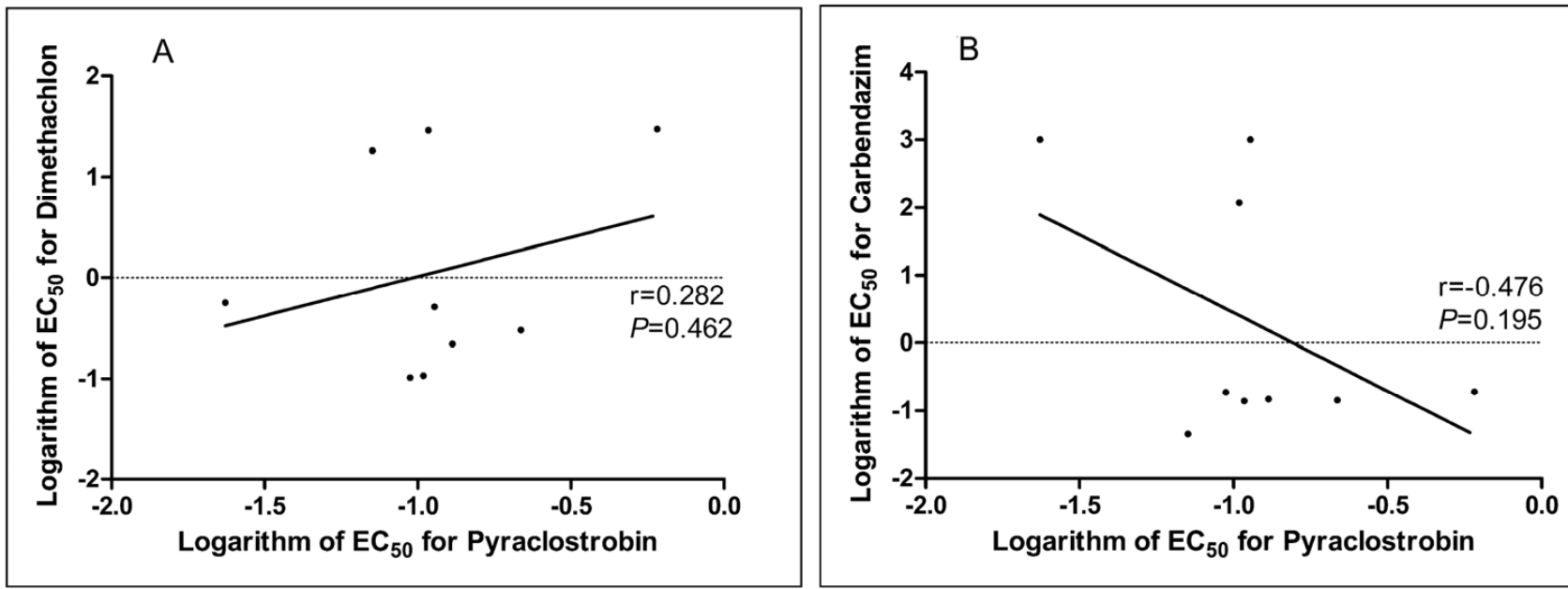

Fig. 2. Pearson correlation coefficients $(r)$ between sensitivity to pyraclostrobin and sensitivity to $\mathbf{A}$, dimethachlon and $\mathbf{B}$, carbendazim.

Table 2. Preventive and curative activity of pyraclostrobin against Sclerotinia sclerotiorum on detached leaves of oilseed rape

\begin{tabular}{|c|c|c|c|c|c|c|c|c|}
\hline \multirow[b]{3}{*}{$\operatorname{Conc}(\mu \mathrm{g} / \mathrm{ml})^{\mathrm{z}}$} & \multicolumn{4}{|c|}{ Preventive control efficacy $(\%)$} & \multicolumn{4}{|c|}{ Curative control efficacy $(\%)^{y}$} \\
\hline & \multicolumn{2}{|c|}{$24 \mathrm{~h}$ after inoculation } & \multicolumn{2}{|c|}{$48 \mathrm{~h}$ after inoculation } & \multicolumn{2}{|c|}{$24 \mathrm{~h}$ after application } & \multicolumn{2}{|c|}{$48 \mathrm{~h}$ after application } \\
\hline & Pyra & Carb & Pyra & Carb & Pyra & Carb & Pyra & Carb \\
\hline 10 & 100.00 & $49.99 *$ & 92.59 & $44.95^{*}$ & 19.39 & 20.54 & 31.54 & 28.83 \\
\hline 20 & 100.00 & $56.97 *$ & 100.00 & $52.36^{*}$ & 58.07 & $23.74 *$ & 74.44 & $39.05 *$ \\
\hline 40 & 100.00 & 82.14 & 100.00 & $69.55^{*}$ & 66.91 & $44.79^{*}$ & 77.31 & 63.83 \\
\hline
\end{tabular}

${ }^{\mathrm{x}}$ Pyra $=$ pyraclostrobin, Carb $=$ carbendazim, and $*$ indicates the control efficacy of carbendazim was significantly different from that of pyraclostrobin under the same conditions according to Student's test at $P=0.05$. Control efficacy data were transformed by arcsine square root method before statistical analysis.

${ }^{y}$ For curative control efficacy, fungicides were applied $24 \mathrm{~h}$ after inoculation.

${ }^{\mathrm{z}}$ Concentration.

Table 3. Preventive and curative activity of pyraclostrobin against Sclerotinia sclerotiorum in pot experiments ${ }^{\mathrm{x}}$

\begin{tabular}{|c|c|c|c|c|c|c|c|c|}
\hline \multirow[b]{3}{*}{$\operatorname{Conc}(\mu \mathrm{g} / \mathrm{ml})^{\mathrm{z}}$} & \multicolumn{4}{|c|}{ Preventive control efficacy $(\%)$} & \multicolumn{4}{|c|}{ Curative control efficacy $^{y}$} \\
\hline & \multicolumn{2}{|c|}{$48 \mathrm{~h}$ after inoculation } & \multicolumn{2}{|c|}{$72 \mathrm{~h}$ after inoculation } & \multicolumn{2}{|c|}{$48 \mathrm{~h}$ after application } & \multicolumn{2}{|c|}{$72 \mathrm{~h}$ after application } \\
\hline & Pyra & Carb & Pyra & Carb & Pyra & Carb & Pyra & Carb \\
\hline 5 & 79.76 & $57.94 *$ & 61.25 & $42.70^{*}$ & 29.79 & 37.97 & 18.39 & 17.34 \\
\hline 15 & 100.00 & $76.19 *$ & 76.67 & 62.92 & 44.04 & 45.74 & 32.12 & 26.60 \\
\hline 45 & 100.00 & 100.00 & 100.00 & $74.90 *$ & 80.99 & 63.64 & 50.05 & 57.96 \\
\hline
\end{tabular}

${ }^{\mathrm{x}}$ Pyra $=$ pyraclostrobin, Carb $=$ carbendazim, and $*$ indicates the control efficacy of carbendazim was significantly different from that of pyraclostrobin under the same conditions according to Student's test at $P=0.05$. Control efficacy data were transformed by arcsine square root method before statistical analysis.

${ }^{\text {y }}$ For curative control efficacy, fungicides were applied $24 \mathrm{~h}$ after inoculation.

${ }^{\mathrm{z}}$ Concentration. 
gene leading to an amino-acid change from glycine to alanine at position 143 (G143A) may confer high levels of resistance to QoI fungicide (34). The Fungicide Resistance Action Committee (FRAC) categorized QoI fungicides as high risk for resistance development (10). QoI fungicides were first commercially available in 1996 and within, 2 to 4 years of commercial marketing, resistance was detected in field populations of several pathogen species (11). Thus far, there have been numerous reports of QoI fungicide resistance in a variety of fungal plant pathogens, including Alternaria solani $(23,24)$, Botrytis cinerea $(12,15)$, Cercospora beticola (Sacc.) (20), Erysiphe graminis f. sp. tritici (8), Magnaporthe grisea (1), M. oryzae (18), Pyricularia grisea (14), and Ustilago maydis (42).

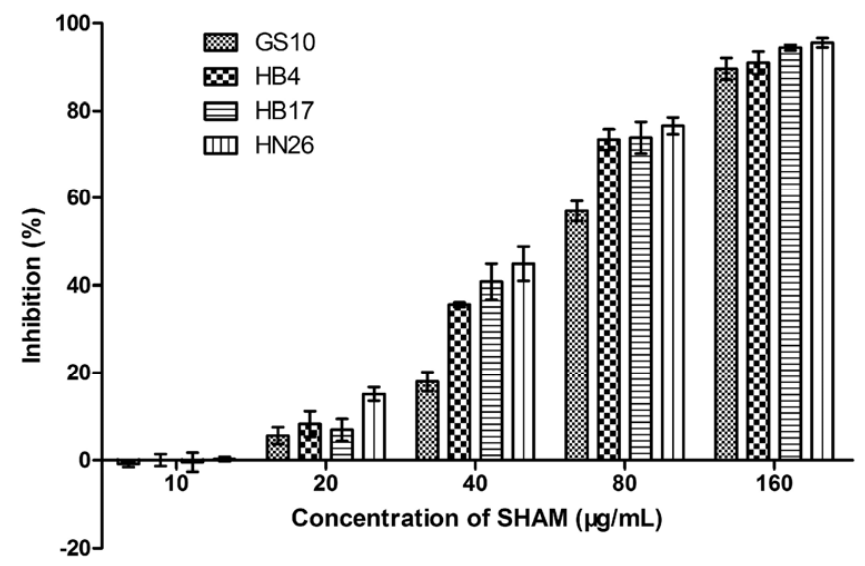

Fig. 3. Mycelial growth inhibitions of Sclerotinia sclerotiorum by increasing concentrations of salicylhydroxamic acid (SHAM) on potato dextrose agar media. The error bar indicates standard deviation (SD) of three replicates. Calculated SHAM effective concentration for $50 \%$ inhibition of mycelial growth values for isolates HN26, HB17, HB4, and GS10 were 44.40, 51.80, 54.43, and $68.68 \mu \mathrm{g} / \mathrm{ml}$, respectively.
Pyraclostrobin has been reported to be an excellent systemic fungicide with both preventive and curative activities on a wide range of fungal plant pathogens (38). The present study demonstrated that the preventive control efficacy was higher than that of carbendazim but the curative control efficacy of pyraclostrobin applied $24 \mathrm{~h}$ after inoculation was similar to that of carbendazim. The spray volume in this study was higher than commonly applied in the field, so that every leaf could be sprayed until runoff. However, the concentrations used in this study were much lower than the recommended label rate for pyraclostrobin against other pathogens. In the case of Sphaerotheca cucurbitae on cucumber, the recommended label rate in China is about 80 to $250 \mu \mathrm{g} / \mathrm{ml}$. Because the biochemical mode of action of pyraclostrobin is different from those of carbendazim and dimethachlon, there is no positive cross-resistance between pyraclostrobin and either carbendazim or dimethachlon, as demonstrated in this study. Therefore, pyraclostrobin should be an excellent fungicide candidate for management of Sclerotinia sclerotiorum resistant to carbendazim or dimethachlon. Nevertheless, great care should be taken and precautious measures must be adopted to avoid or delay the development of resistance to pyraclostrobin because $S$. sclerotiorum has shown a high potential for resistance development to commonly used fungicides such as dimethachlon $(19,40,41)$ and, especially, carbendazim $(17,25,39)$. According to the guidelines recommended by FRAC for management of fungicide resistance, judicious fungicide application strategies include (i) not using a single fungicide exclusively, (ii) restricting the number of applications per season, and (iii) applying fungicides as preventative protectants rather than as eradicants (6). Integrated pathogen management tactics such as disease-resistant plant varieties, biological control agents, crop rotations, and mixtures or rotations of pyraclostrobin with other fungicides with different modes of action should be implemented to delay or prevent the possible development of pyraclostrobin resistance in S. sclerotiorum.

The common practice of adding SHAM to artificial media in assay of QoI fungicide sensitivity is for inhibition of AOX, which is

Table 4. Sclerotinia sclerotiorum effective concentration for $50 \%$ inhibition of mycelial growth $\left(\mathrm{EC}_{50}\right)$ values for pyraclostrobin with and without salicylhydroxamic acid (SHAM)

\begin{tabular}{|c|c|c|c|c|c|}
\hline \multirow[b]{2}{*}{ Isolate } & \multicolumn{2}{|c|}{ Pyraclostrobin with SHAM ${ }^{w}$} & \multicolumn{2}{|c|}{ Pyraclostrobin without SHAM } & \multirow[b]{2}{*}{ Significance $^{y}$} \\
\hline & $\mathbf{E C}_{50}(\mu \mathrm{g} / \mathrm{ml})$ & $95 \%$ CI of $\mathrm{EC}_{50}{ }^{\mathrm{x}}$ & $\mathrm{EC}_{50}(\mu \mathrm{g} / \mathrm{ml})$ & $95 \%$ CI of $\mathrm{EC}_{50}{ }^{\mathrm{x}}$ & \\
\hline HN1 & 0.0156 & $0.0107-0.0226$ & 0.0632 & $0.0462-0.0865$ & * \\
\hline HN26 & 0.0175 & $0.0053-0.0580$ & 0.0993 & $0.0637-0.1548$ & * \\
\hline HB4 & 0.0253 & $0.0183-0.0351$ & 0.0680 & $0.0527-0.0877$ & * \\
\hline HB17 & 0.0166 & $0.0059-0.0467$ & 0.0524 & $0.0316-0.0869$ & $\ldots$ \\
\hline GS22 & 0.0140 & $0.0092-0.0215$ & 0.0465 & $0.0419-0.0517$ & $*$ \\
\hline GS10 & 0.0216 & $0.0107-0.0435$ & 0.0438 & $0.0307-0.0624$ & \\
\hline SX5 & 0.0134 & $0.0111-0.0163$ & 0.0362 & $0.0347-0.0378$ & * \\
\hline SX23 & 0.0145 & $0.0062-0.0340$ & 0.0380 & $0.0279-0.0518$ & $\ldots$ \\
\hline HLJ23 & 0.0126 & $0.0073-0.0217$ & 0.0646 & $0.0392-0.1065$ & $*$ \\
\hline HLJ13 & 0.0216 & $0.0116-0.0403$ & 0.0754 & $0.0506-0.1126$ & $*$ \\
\hline Average & 0.0173 & $\ldots$ & 0.0587 & $\ldots$ & $P<0.0001^{\mathrm{z}}$ \\
\hline
\end{tabular}

${ }^{\mathrm{w}}$ Final concentration of SHAM in potato dextrose agar media was $20 \mu \mathrm{g} / \mathrm{ml}$.

${ }^{\mathrm{x}}$ The 95\% confidence interval (CI) was calculated by statistical software package Data Processing System (ver. 7.05; Hangzhou RuiFeng Information Technology Co. Ltd.).

${ }^{\mathrm{y}} \mathrm{An}$ asterisk (*) denotes that difference in $\mathrm{EC}_{50}$ values between pyraclostrobin with and without SHAM was statistically significant. The criterion for significant difference in $\mathrm{EC}_{50}$ values was according to Robertson and Preisler (26), as follows: the two $\mathrm{EC}_{50}$ values were considered significantly different if there was no overlap for $95 \%$ CIs of the two $\mathrm{EC}_{50}$ values.

${ }^{\mathrm{z}}$ Student's paired $t$ test was employed for significant difference in the mean $\mathrm{EC}_{50}$ values of the 10 isolates between pyraclostrobin with and without $\mathrm{SHAM}$.

Table 5. Potentiation of control efficacy of pyraclostrobin against Sclerotinia sclerotiorum in pot experiments by SHAM

\begin{tabular}{lcccr}
\hline & \multicolumn{4}{c}{ Control efficacy $\mathbf{( \% )}$} \\
\cline { 2 - 4 } Isolate & SHAM $(\mathbf{2 0} \boldsymbol{\mu g} / \mathbf{m l})$ & Pyraclostrobin $(\mathbf{1 0} \boldsymbol{\mu g} / \mathbf{m l})$ & SHAM + Pyraclostrobin $(\mathbf{2 0}+\mathbf{1 0} \boldsymbol{\mu g} / \mathbf{m l})$ & Co-toxicity factor $^{\mathbf{z}}$ \\
\hline SX23 & 9.32 & 42.78 & 81.28 & 68.94 \\
HN26 & -9.14 & 45.97 & 82.90 & 102.04 \\
HN18 & 8.95 & 46.33 & 85.52 & 67.25 \\
HB4 & 7.62 & 62.11 & 47.19 & -27.40 \\
\hline
\end{tabular}

${ }^{\mathrm{z}}$ Co-toxicity factor greater than 20 denotes synergism and co-toxicity factor lower than -20 denotes antagonism. 
presumed to be inhibited during infections by plant secondary metabolites such as flavonoid (35). If fungal AOX can be completely inhibited in planta, then it is incomprehensible that SHAM can significantly increase control efficacies of pyraclostrobin, as has been shown in the present study. One possible explanation is that the secondary metabolites of oilseed rape might not, or at least not completely, inhibit AOX of $S$. sclerotiorum during infections. Up to the present, no direct biochemical evidence has been reported that AOX can be inhibited by secondary metabolites of oilseed rape during infections. Another possible reason for the significantly increased control efficacy may be the direct toxicity of SHAM on S. sclerotiorum. Studies by $\mathrm{Xu}$ et al. (37) demonstrated that AOX is involved in the regulation of growth, development, and resistance to oxidative stress of $S$. sclerotiorum. Nonetheless, the direct toxic effects of SHAM at $20 \mu \mathrm{g} / \mathrm{ml}$ or higher concentrations on $S$. sclerotiorum may not be caused totally by the inhibition of AOX because AOX is only the branched oxidase to the core respiratory pathway (35). The toxic effect of SHAM may be partially due to its inhibitory activity on other essential fungal enzymes, such as peroxidase, putative melanogenesis enzymes, esterase, and laccase $(2,30)$. The toxic effect of SHAM has been reported on other fungal pathogens; for example, even at low concentrations, SHAM could suppress mycelial growth of F. effusum on PDA media (28). With respect to the direct effect of SHAM on fungicide sensitivity assays, previous studies also demonstrated that SHAM at $100 \mu \mathrm{g} / \mathrm{ml}$ could decrease $\mathrm{EC}_{50}$ value of the QoI fungicide azoxystrobin against $S$. sclerotiorum but increase $\mathrm{EC}_{50}$ value of the dicarboximide fungicide procymidone $(9,36)$. This evidence indicates that addition of SHAM into artificial media in assay of QoI fungicide sensitivity may lead to inaccurate or erroneous results. Therefore, at least for determination of $S$. sclerotiorum sensitivity to pyraclostrobin based on mycelial growth inhibition on PDA media, SHAM should not be added.

\section{Acknowledgments}

This study was supported by the Special Fund for Agro-scientific Research in the Public Interest (number 201103016). We thank D. Jiang of Huazhong Agricultural University for technical support.

\section{Literature Cited}

1. Avila-Adame, C., and Köller, W. 2003. Characterization of spontaneous mutants of Magnaporthe grisea expressing stable resistance to the Qo inhibiting fungicide azoxystrobin. Curr. Genet. 42:332-338.

2. Baek, H. S., Rho, H. S., Yoo, J. W., Ahn, S. M., Lee, J. Y., Lee, J., Kim, M., Kim, D. H., and Chang, I. S. 2008. The inhibitory effect of new hydroxamic acid derivatives on melanogenesis. Bull. Korean Chem. Soc. 29:43-46.

3. Bartlett, D. W., Clough, J. M., Godwin, J. R., Hall, A., Hamer, M., and ParrDobrzanski, B. 2002. The strobilurin fungicides. Pest Manage. Sci. 58:649662.

4. Boland, G. J., and Hall, R. 1994. Index of plant hosts of Sclerotinia sclerotiorum. Can. J. Plant Pathol. 16:93-108.

5. Bolton, D. M., Thomma, B. P., and Nelson, B. D. 2006. Sclerotinia sclerotiorum (Lib.) de Bary: Biology and molecular traits of a cosmopolitan pathogen. Mol. Plant Pathol. 7:1-16.

6. Brent, K. J., and Hollomon, D. W. 2007. Fungicide resistance in crop pathogens: How can it be managed? FRAC Monograph No. 1. 2nd ed. Brussels. http://www.frac.info/publication/anhang/FRAC_Mono1_2007_100dpi.pdf

7. Brent, K. J., and Hollomon, D. W. 2007. Fungicide resistance: The assessment of risk. FRAC Monograph No. 2. Second Ed. Brussels. http://www.frac.info/publication/anhang/FRAC_Mono2_2007.pdf

8. Chin, K. M., Chavaillaz, D., Kaesbohrer, M., Staub, T., and Felsenstein, F. G. 2001. Characterizing resistance risk of Erysiphe graminis f. sp. tritici to strobilurins. Crop Prot. 20:87-96.

9. Duan, Y. B., Liu, S. M., Ge, C. Y., Feng. X. J., Chen, C. J., and Zhou, M. G. 2012. In vitro inhibition of Sclerotinia sclerotiorum by mixtures of azoxystrobin, SHAM and thiram. Pestic. Biochem. Physiol. 103:101-107.

10. FRAC (Fungicide Resistance Action Committee). 2014. FRAC Code List 2014: Fungicide sorted by mode of action. http://www.frac.info/publication/ anhang/2014\%20FRAC\%20Code\%20List.pdf

11. Gisi, U., Sierotzki, H., Cook, A., and McCaffery, A. 2002. Mechanisms influencing the evolution of resistance to QoI inhibitor fungicides. Pest Manage. Sci. 58:859-867.

12. Ishii, H., Fountaine, J., Chung, W., Kansako, M., Nishimura, K., Takahashi, K., and Oshima, M. 2009. Characterization of QoI-resistant field isolates of Botrytis cinerea from citrus and strawberry. Pest Manage. Sci. 65:916-922.
13. Kaneko, I., and Ishii, H. 2009. Effect of azoxystrobin on activities of antioxidant enzymes and alternative oxidase in wheat head blight pathogens Fusarium graminearum and Microdochium nivale. J. Gen. Plant Pathol. 75:388-398.

14. Kim, Y. S., Dixon, E. W., Vincelli, P., and Farman, M. L. 2003. Field resistance to strobilurin (QoI) fungicides in Pyricularia grisea caused by mutations in the mitochondrial cytochrome b gene. Phytopathology 93:891900.

15. Kim, Y. K., and Xiao, C. L. 2011. Stability and fitness of pyraclostrobinand boscalid-resistant phenotypes in field isolates of Botrytis cinerea from apple. Phytopathology 101:1385-1391.

16. Kosman, E., and Cohen, Y. 1996. Procedures for calculating and differentiating synergism and antagonism in action of fungicide mixtures. Phytopathology 86:1263-1272.

17. Kuang, J., Wang, J. X., and Zhou, M. G. 2011. Monitoring carbendazim and dimethachlon resistance of Sclerotinia sclerotiorum obtained from the blight stems of rape in Jiangsu Province. Chin. Agric. Sci. Bull. 27:285-291.

18. Ma, B., and Uddin, W. 2009. Fitness and competitive ability of an azoxystrobin-resistant G143A mutant of Magnaporthe oryzae from perennial ryegrass. Plant Dis. 93:1044-1049.

19. Ma, H. X., Feng, X. J., Chen, Y., Chen, C. J., and Zhou, M. G. 2009. Occurrence and characterization of dimethachlon insensitivity in Sclerotinia sclerotiorum in Jiangsu Province of China. Plant Dis. 93:36-42.

20. Malandrakis, A. A., Markoglou, A. N., Nikou, D. C., Vontas, J. G., and Ziogas, B. N. 2006. Biological and molecular characterization of laboratory mutants of Cercospora beticola resistant to Qo inhibitors. Eur. J. Plant Pathol. 116:155-166.

21. Markoglou, A. N., Malandrakis, A. A., Vitoratos, A. G., and Ziogas, B. N. 2006. Characterization of laboratory mutants of Botrytis cinerea resistant to QoI fungicides. Eur. J. Plant Pathol. 115:149-162.

22. Pan, Y. L. 1998. The resistance of Sclerotinia sclerotiorum of rape to carbendazim and its management. Jiangsu J. Agric. Sci. 14:159-163.

23. Pasche, J. S., Piche, L. M., and Gudmestad, N. C. 2005. Effect of the F129L mutation in Alternaria solani on fungicides affecting mitochondrial respiration. Plant Dis. 89:269-278.

24. Pasche, J. S., Wharam, C. M., and Gudmestad, N. C. 2004. Shift in sensitivity of Alternaria solani in response to QoI fungicides. Plant Dis. 88:181187.

25. Qi, Y. X., Chen, F. X., Su, X. Y., Ding, K. J., Yu, J., and Jiang, M. S. 2006. Monitoring on carbendazim resistance of Sclerotinia sclerotiorum obtained from the blight stems of rape in Anhui Province. Chin. Agric. Sci. Bull. 22:371-373.

26. Robertson, J. L., and Preisler, H. K. 1992. Pesticide Bioassays with Arthropods. CRC Press, Inc., Boca Raton, FL.

27. Russell, P. E. 2004. Sensitivity baselines in fungicide resistance research and management. FRAC Monograph No. 3. Brussels. http://www.frac.info/ publication/anhang/monograph3.pdf

28. Seyran, M., Brenneman, T. B., and Stevenson, K. L. 2010. In vitro toxicity of alternative oxidase inhibitors salicylhydroxamic acid and propyl gallate on Fusicladium effusum. J. Pestic. Sci. 83:421-427.

29. Shi, Z. Q., Zhou, M. G., and Ye, Z. Y. 2000. Resistance of Sclerotinia sclerotiorum to carbendazim and dimethachlon. Chin. J. Oil Crop Sci. 22:5457.

30. Tsukamoto, K., Itakura, H., Sato, K., Fukuyama, K., Miura, S., Takahashi, S., Ikezawa, H., and Hosoya, T. 1999. Binding of salicylhydroxamic acid and several aromatic donor molecules to Arthromyces ramosus peroxidase, investigated by x-ray crystallography, optical difference spectroscopy, NMR relaxation, molecular dynamics, and kinetics. Biochemistry 38:1255812568 .

31. Vincelli, P. 2002. QoI (Strobilurin) fungicides: Genefits and risks. The Plant Heath Instructor. doi:10.1094/PHI-I-2002-0809-02 http://www.apsnet.org/ edcenter/advanced/topics/Pages/StrobilurinFungicides.aspx

32. Walker, A., Auclair, C., Gredt, M., and Leroux, P. 2009. First occurrence of resistance to strobilurin fungicides in Microdochium nivale and Microdochium majus from French naturally infected wheat grains. Pest Manage. Sci. 65:906-915.

33. Wang, Y., Hou, Y. P., Chen, C. J., and Zhou, M. G. 2014. Detection of resistance in Sclerotinia sclerotiorum to carbendazim and dimethachlon in Jiangsu Province of China. Australas. Plant Pathol. 43:307-312.

34. Wise, K. A., Bradley, C. A., Pasche, J. S., Gudmestad, N. C., Dugan, F. M. and Chen, W. 2008. Baseline sensitivity of Ascochyta rabiei to azoxystrobin, pyraclostrobin, and boscalid. Plant Dis. 92:295-300.

35. Wood, P. M., and Hollomon, D. W. 2003. A critical evaluation of the role of alternative oxidase in the performance of strobilurin and related fungicides acting at the Qo site of Complex III. Pest Manage. Sci. 59:499-511.

36. Xu, T., Wang, Y. T., Liang, W. S., Yao, F., Li, Y. H., Li, D. R., Wang, H., and Wang, Z. Y. 2013. Involvement of alternative oxidase in the regulation of sensitivity of Sclerotinia sclerotiorum to the fungicides azoxystrobin and procymidone. J. Microbiol. 51:352-358.

37. Xu, T., Yao, F., Liang, W. S., Li, Y. H., Li, D. R., Wang, H., and Wang, Z. Y. 2012. Involvement of alternative oxidase in the regulation of growth, development, and resistance to oxidative stress of Sclerotinia sclerotiorum. J. Microbiol. 50:594-602.

38. Yang, L. J., and Bai, Y. L. 2012. Strobilurin fungicide-Pyraclostrobin. 
Modern Agrochemicals 11:46-56.

39. Zhang, X. L., Sun, X. M., Zhang, G. F., Yi, H. J., and Qiang, Y. 2003. Preliminary report on the monitoring of the resistance of Sclerotinia sclerotiorum to carbendazim and its integrated management. Chin. J. Pestic. Sci. Adm. 24:18-22.

40. Zhou, F., Zhang, X. L., Li, J. L., and Zhu, F. X. 2014. Dimethachlon resistance in Sclerotinia sclerotiorum in China. Plant Dis. 98:1221-1226.
41. Zhou, F., Zhu, F. X., Zhang, X. L., and Zhang, A. S. 2014. First report of dimethachlon resistance in field isolates of Sclerotinia sclerotiorum on oilseed rape in Shaanxi Province of northwestern China. Plant Dis. 98:568

42. Ziogas, B. N., Markoglou, A. N., and Tzima, A. 2002. A non-Mendelian inheritance of resistance to strobilurin fungicides in Ustilago maydis. Pest Manage. Sci. 58:908-916 